Cipango $\begin{aligned} & \text { Cipango } \\ & \text { Cahiers d'études japonaises }\end{aligned}$

$15 \mid 2008$

Guerre, colonialisme et commémoration

\title{
Les sources d'inspiration et les influences dans la musique de Yoshihisa Taira
}

\section{Chen Hui-Mei}

\section{OpenEdition}

1 Journals

\section{Édition électronique}

URL : https://journals.openedition.org/cipango/449

DOI : $10.4000 /$ cipango.449

ISSN : 2260-7706

Éditeur

INALCO

\section{Édition imprimée}

Date de publication : 1 janvier 2008

Pagination : 294-297

ISBN : 978-2-85831-177-4

ISSN : $1164-5857$

\section{Référence électronique}

Chen Hui-Mei, "Les sources d'inspiration et les influences dans la musique de Yoshihisa Taira ", Cipango [En ligne], 15 | 2008, mis en ligne le 14 novembre 2011, consulté le 30 juin 2021. URL : http:// journals.openedition.org/cipango/449; DOI : https://doi.org/10.4000/cipango.449

Ce document a été généré automatiquement le 30 juin 2021.

\section{(c) (7) (8)}

Cipango est mis à disposition selon les termes de la Licence Creative Commons Attribution - Pas d'Utilisation Commerciale 4.0 International. 


\title{
Les sources d'inspiration et les influences dans la musique de Yoshihisa Taira
}

\author{
Chen Hui-Mei
}

\section{RÉFÉRENCE}

Les sources d'inspiration et les influences dans la musique de Yoshihisa Taira, Thèse de doctorat soutenue par Chen Hui-Mei (Paris IV-Sorbonne, 2007) sous la direction de M.

Battier.

1 Le point de départ de cette recherche est la force irrésistible qui se dégage de la musique de Taira Yoshihisa 平義久. Compositeur né à Tōkyō en 1937, il séjourna à Paris depuis son arrivée en 1966 et s'y est éteint en 2005. Avec une œuvre originale de qualité et un catalogue de quatre-vingts opus environ, la musique de Taira lui a valu plusieurs grands prix. Ses œuvres ont été créées dans le cadre des principaux festivals et des institutions de musique contemporaine, en France comme à l'étranger, et elles sont appréciées par le public comme par les interprètes.

Contrairement à d'autres compositeurs japonais, Taira n'a jamais écrit pour les instruments traditionnels, ni appliqué la théorie musicale de son pays d'origine, pourtant sa musique est souvent décrite comme très japonaise. C'est en cherchant les particularités et l'originalité de la musique de Taira que nous tâchons de comprendre pourquoi cette musique donne une impression très japonaise, aussi en quoi elle se différencie et en quoi elle est comparable à la musique d'autres compositeurs. Nos préoccupations consistent à chercher de quelle manière les sources d'inspiration et les influences réciproques de deux cultures coexistent dans la musique de ce compositeur.

3 Puisque les méthodes analytiques musicales traditionnelles qui s'appuient principalement sur les paramètres harmoniques, mélodiques ou formels ne s'adaptent pas tout à fait à une œuvre musicale de notre temps, nous appliquons parallèlement 
d'autres méthodologies en essayant de tenir comptes des aspects culturels, philosophiques, historiques, psychologiques et métaphoriques.

4 Cette thèse est divisée en trois parties. Consacrée à l'histoire, la première partie comporte deux chapitres: le premier commence par un aperçu historique de l'introduction de la musique occidentale au Japon afin de comprendre dans quelle condition cette musique a été importée dans ce pays avant d'arriver à une présentation de la situation florissante de la création musicale japonaise de nos jours. Le deuxième chapitre traite l'histoire personnelle de Taira, depuis son enfance jusqu'à ses années de formation, au Japon et en France.

5 Après l'histoire, les problématiques de cette recherche sont abordées dans la deuxième partie qui est divisée en trois chapitres. Le chapitre 3 présente quelques caractéristiques de la musique de Taira. Souvent chez ce compositeur, les sources d'inspiration jouent non seulement un rôle initiateur qui suggère un projet d'œuvre musicale, mais aussi reflète sa pensée musicale et philosophique. Ces sources du début se transforment en réalisation concrète et se manifestent dans le choix des instruments, les structures formelles et l'organisation du discours musical. Le contact avec les grands maîtres, puis avec ses condisciples et ses compatriotes, ses amis compositeurs : les sources d'inspirations et les références dans la musique de Taira sont multiples. Le chapitre 4 tente d'en saisir quelques-unes. La discussion avec ses amis a aussi inspiré Taira. De nombreuses notions esthétiques, compositionnelles, philosophiques chez lui sont en rapport avec celles de ses amis et de compositeurs occidentaux ou asiatiques. Le chapitre 5 clôt la deuxième partie en présentant quelques-uns de ces compositeurs prédécesseurs ou contemporains de Taira.

6 La troisième partie est consacrée à une analyse musicale centrée principalement sur trois de ses œuvres les plus représentatives. C'est en analysant ces partitions sélectionnées que nous tentons de montrer comment les sources d'inspiration et les influences mentionnées précédemment se manifestent dans la musique de Taira. Le chapitre 6 traite du cycle des Hiérophonies composé entre 1969 et 1975, qui est le point de départ de la carrière de Taira. Cette œuvre comporte les germes de presque tout le vocabulaire significatif du langage musical du compositeur. Le chapitre 7 examine Méditations pour orchestre (1977). Le choix de cette œuvre se justifie non seulement par l'ampleur de sa formation, la maturité de son écriture, l'importance de sa place dans l'ensemble du catalogue, mais aussi par l'intérêt de montrer la continuité de la pensée musicale chez Taira. A ceci s'ajoute encore la volonté du compositeur qui souhaitait voir cette œuvre monumentale être analysée. Le chapitre 8 qui met fin à cette troisième partie est réservé à Retour pour soprano et ensemble instrumental, créé en juin 2003, peu avant le décès du musicien, pour ainsi montrer la continuité, l'évolution des pensées musicales et leur réalisation au cours de différentes époques créatrices. Nous commençons chaque analyse par une présentation du contexte de création, une description de l'œuvre et un petit tableau de la division en sections, avant d'aborder d'autres paramètres d'analyse en espérant fournir une image plus précise de l'œuvre.

Certes la créativité, l'authenticité musicale dans l'ensemble de l'œuvre de Taira est née de la confrontation de deux cultures très différentes, voire opposées: l'Occident ne célèbre pas les mêmes valeurs philosophiques, spirituelles et sociales que le Japon, pourtant les deux mondes cohabitent en lui. Toutefois nous ne voulons pas l'écraser sous son appartenance culturelle en prétendant que sa musique relève d'une prétendue esthétique japonaise. Si Taira est finalement très marqué par sa culture japonaise, les 
influences de compositeurs européens, et en particulier français comme Debussy, Messiaen, Dutilleux et Jolivet, ne restent pas moins présentes. S'il existe chez Taira une "japonitude», elle est profondément intériorisée. Cela concerne peut-être le raffinement des timbres ou la coexistence de la subtilité et l'énergie.

Composer de la musique occidentale contemporaine reste toujours une gageure pour les compositeurs d'origine asiatique, car on en attend d'eux une musique qui intègre des éléments traditionnels. Pourtant un musicien qui reçoit une formation musicale à l'occidentale ignore très souvent la musique de son pays : c'est très fréquent chez les compositeurs asiatiques. Pendant des décennies, nombreux sont ceux qui ont cherché le dialogue entre deux cultures : l'une vient de leur origine et l'autre de leur formation. Ce dialogue apparaît évidemment comme un vieux thème éculé si l'on ne se donne pas la peine de le pratiquer ni de l'observer à l'œuvre, c'est-à-dire comme un dialogue constitutif de musiques qui réussissent pleinement cette intégration. C'est pour cela que nous avons proposé d'examiner en détail quelques partitions sélectionnées et tenté de suivre les traces de cette intégration afin d'illustrer comment ce compositeur japonais, avec sa sensibilité individuelle, a trouvé sa voie originale en composant avec un langage musical fondé sur une esthétique qui ouvre à l'évidence un nouveau et immense champ d'investigation. 